\title{
Effect of tracheobronchial suction on respiratory resistance in intubated preterm babies
}

\author{
A PRENDIVILLE, A THOMSON, AND M SILVERMAN \\ Department of Paediatrics and Neonatal Medicine, Royal Postgraduate Medical School, Hammersmith \\ Hospital, London
}

SUMMARY Measurements of the resistance, time constant, and compliance of the respiratory system were made in 25 mechanically ventilated, preterm babies on 32 occasions, using the single breath technique. Patients were classified according to the level of respiratory resistance by reference to a population of 36 babies studied over the first two days of life before airway secretions had become apparent. There was a highly significant fall in both the resistance and time constant measurements after tracheobronchial suction or lavage for infants whose pretreatment values of resistance were greater than the reference mean. When tracheobronchial toilet was effective in removing secretions the changes in resistance and time constant values were again significant. No changes in compliance values were noted. Severe but clinically inapparent mucous obstruction of the airways was revealed in two infants by a progressively rising respiratory system resistance during continuous monitoring. The need for and efficacy of tracheobronchial suction and lavage could be determined by such techniques for monitoring theo resistance of the respiratory system during mechanical ventilation.

Routine tracheobronchial suction and lavage are carried out during mechanical ventilation in many neonatal intensive care units. Neither the indications for beginning these procedures nor their optimal frequency has been established. There is now growing evidence to suggest that the adverse side effects of suction or lavage, such as transient bacteraemia, ${ }^{1}$ increased intracranial pressure, ${ }^{2}$ diminished mucociliary transport, ${ }^{3}$ and airway inflammation, ${ }^{4}$ may be considerable. In addition, there exists the obvious disturbance to ventilation during the procedure.

In the extreme situation where the airway is almost totally occluded by mucus endotracheal suction produces obvious and rapid clinical benefit. Lesser degrees of mucous obstruction are, however, notoriously difficult to identify in the usual clinical setting. Moreover, using existing techniques, the beneficial effects of chest physiotherapy and suction have been difficult to establish. ${ }^{56}$

We therefore set out to try and predict the optimal timing and mechanical benefits of tracheobronchial suction or lavage, by using single breath measurements of lung mechanics ${ }^{7}$ to measure the compliance, resistance, and time constant of the respiratory system before and after tracheobron- chial toilet in infants with and without clinically obvious airways secretions.

\section{Patients and methods}

Patients. To establish a reference range of values of resistance of the respiratory system in intubated, mechanically ventilated, preterm babies 36 babies were studied towards the end of day one of life and 21 babies on day 2 of life. Data collected on days 1 and 2 of life were used because at this stage there was no evidence of appreciable secretions. Mean (SD) gestational age was $29.5(3.6)$ weeks and mean (SD) birth weight $1370(740) \mathrm{g}$. The babies were divided into two clinical groups:

(1) 25 babies with presumed hyaline membrane disease defined clinically and radiologically;

(2) 11 babies ventilated for non-respiratory or non-cardiac problems who had no clinical or radiological evidence of respiratory disease.

The study group consisted of 25 babies of mean (SD) gestational age 30.6 (4.9) weeks and mean (SD) birth weight $1440(700) \mathrm{g}$. They were studied on 32 occasions after the second day of life. All these babies were receiving routine ventilator care, including regular tracheobronchial suction. Some in 
whom there was a particular clinical indicationthat is, large amounts of secretions-were being given additional bronchial lavage. The tests were timed to coincide with routine suction or lavage procedures.

Lung mechanics. All babies had been intubated with a 2.5-3.5 Coles tube (Portex) and ventilated with a pressure limited time cycled ventilator (Vickers Neovent or SLE Newborn). The rate of ventilation was increased until spontaneous respiratory effort was abolished as judged by a uniform run of inspiratory and expiratory volume plateaux in response to the ventilator pressure plateaux. Endotracheal tube gas flow was measured using a double pneumotachograph system ${ }^{8}$ linear to at least 12 $1 /$ min placed in the bias flow pathway on either side of the infant's endotracheal tube. The pneumotachographs and their transducers (Validyne MP45) were carefully balanced electronically so that the difference between the signals was zero with no flow through the endoctracheal tube. The difference signal during a measurement was thus the baby's tidal flow.

Airway opening pressure was measured at the oral end of the endotracheal tube using an SE Labs 1150 pressure transducer. Flow and pressure signals were fed through an Emma 4001 amplifier system and recorded simultaneously on a UV chart recorder (SE Labs) and after digitisation at $100 \mathrm{~Hz}$ per channel on a microcomputer (Apple II). Flow was passively integrated to give a volume signal on the chart recorder and digitally integrated by microcomputer.

Recordings were taken of airway opening pressure and flow during mechanical ventilation for at least two periods of 30 seconds at 10 minute intervals. The flow and volume signals were displayed on the visual display unit of the microcomputer and the values of flow and volume between $50 \%$ and $97 \%$ of the total expired volume were submitted to linear regression analysis to obtain the slope of the flow-volume relation, which is equivalent to the time constant of the respiratory system under passive conditions. ${ }^{7}$ Compliance of the respiratory system was the ratio of the expired tidal volume, corrected if necessary for incomplete expiration, ${ }^{9}$ to the measured peak to end expiratory difference in airway opening pressure.

From the values of compliance $\left(\mathrm{C}_{\mathrm{rs}}\right)$ and time constant $\left(T_{r s}\right)$, resistance $\left(R_{r s}\right)$ was calculated as follows:

$$
\mathrm{T}_{\mathrm{rs}}=\mathrm{C}_{\mathrm{rs}} \times \mathrm{R}_{\mathrm{rs}} ; \mathrm{R}_{\mathrm{rs}}=\mathrm{T}_{\mathrm{rs}} / \mathrm{C}_{\mathrm{rs}}
$$

Mean values of time constant, compliance, and resistance were derived from analysis of between three and 12 breaths from each 30 second sample.
Mean coefficients of variation of compliance, resistance, and time constant by our technique are $2 \cdot 3 \%, 5 \cdot 7 \%$, and $5 \cdot 7 \%$, respectively. ${ }^{8}$

We did not subtract the endotracheal tube resistance from the measured respiratory system resistance as functionally the endotracheal tube forms part of the infant's airway.

Endotracheal tube suction and lavage. Suction was carried out using a size 6 FG catheter with side hole and end holes (Portex), which was introduced through the endotracheal tube, passed as far as easily possible, and then withdrawn by $0.5 \mathrm{~cm}$. Suction was then applied during withdrawal using negative pressures no more than $40 \mathrm{cmH}_{2} \mathrm{O}$. If secretions were noted in the catheter the procedure was repeated once. The baby was then reconnected to the ventilator and after a period of five minutes at the pre-existing settings the measurements of lung mechanics were repeated.

In the lavage group the same procedure was followed after introducing $0.5 \mathrm{ml}$ normal saline down the endotracheal tube, reconnecting the baby to the ventilator for five breaths, and then following the suction procedure described above except that in this group the procedure was always repeated at least once.

Most measurements were made before and after routine suction or lavage. Occasionally, during the course of a study, values of resistance, which were being continuously updated by computer analysis, were found to be progressively increasing. Appropriate action was taken and in two cases the individual data are described in detail.

Analysis. As the reference values of resistance, compliance, and time constant of the respiratory system on the first two days of life were shown to have a log normal distribution all analysis was performed after logarithmic transformation. Paired $t$ tests were used to compare the before and after suction or lavage. Data from infants with or without obvious secretions were analysed separately.

\section{Results}

In the reference population there was little difference between the mean values of respiratory system resistance of babies with hyaline membrane disease or without respiratory disease on either the first or second days of life. Overall, the combined data gave a mean value of resistance of $195 \mathrm{cmH}_{2} \mathrm{O} / \mathrm{l} / \mathrm{sec}$ (confidence limits 66-562 $\mathrm{cmH}_{2} \mathrm{O} / 1 / \mathrm{sec}$ ) for the reference group of intubated babies during the first two days of life.

When the study group was classified according to 
the pretreatment value of resistance there were striking differences in the effect of endotracheal tube suction and lavage on respiratory system mechanics (Table 1). If the initial resistance was greater than the mean reference value for days one and two of life (Table 1 group D) there was a highly significant drop in resistance and time constant values after suction or lavage, whereas compliance values were unaltered. The changes in resistance were most striking for those groups of infants whose pretreatment resistance was highest (Table 1 groups $\mathrm{A}$ and $\mathrm{B}$ ). When the pretreatment resistance values were less than the mean reference values, however, there was no measurable benefit from suction or lavage. The group as a whole had a highly significant decrease in resistance and time constant after tracheobronchial suction or lavage but no change in compliance (Table 1).

There were pronounced differences in the effect of suction or lavage on lung mechanics, according to whether or not the procedures were effective in removing airway secretions (Table 2, Fig. 1). Simple endotracheal tube suction had a just significant effect on resistance and time constant when obvious secretions were obtained. When secretions were obtained by bronchial lavage on the other hand resistance fell markedly along with time constant. After ineffective bronchial lavage there was a

Table 1 Effects of endotracheal suction or lavage according to pretreatment total respiratory resistance. Values are mean (95\% confidence limits) for pre- and post-treatment

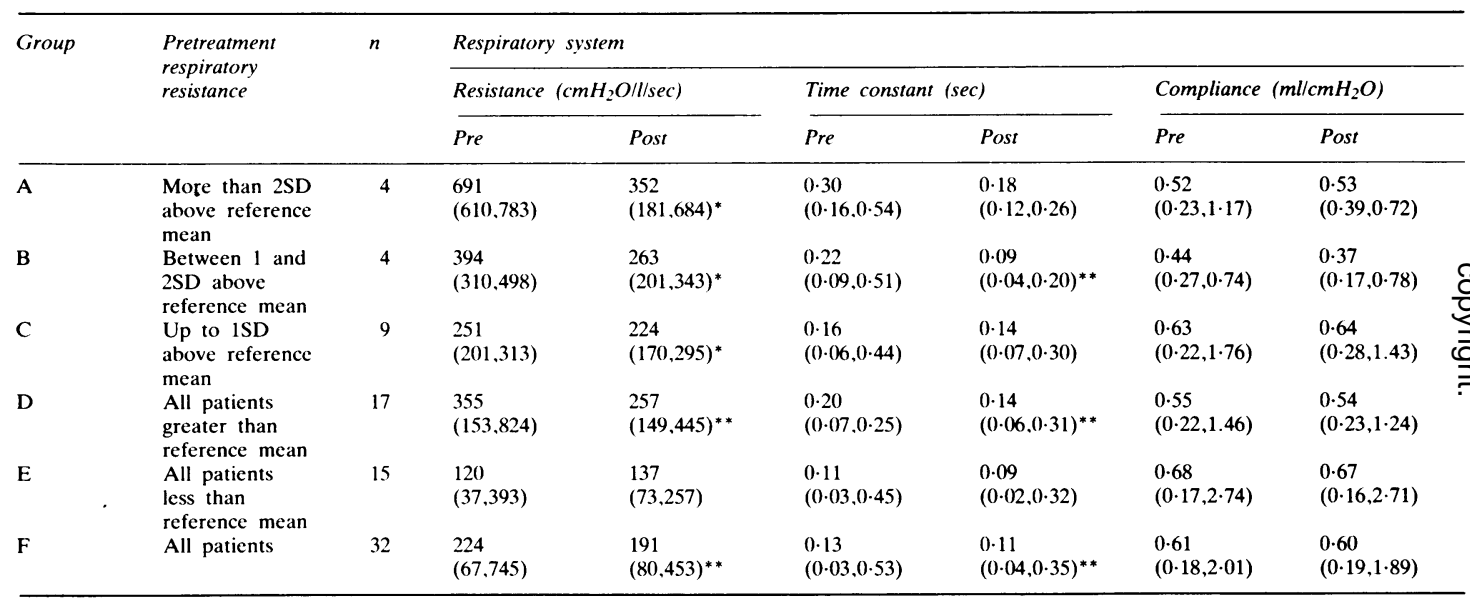

${ }^{*} \mathrm{p}<0 \cdot 05,{ }^{* *} \mathrm{p}<0 \cdot 01$, by $t$ test. Other differences not significant. Log values of respiratory system resistance, time constant, compliance were used in the statistical analysis.

Table 2 Effects of endotracheal suction or lavage according to presence or absence of obvious mucus secretions. Values are mean ( $95 \%$ confidence limits) for pre- and post-treatment

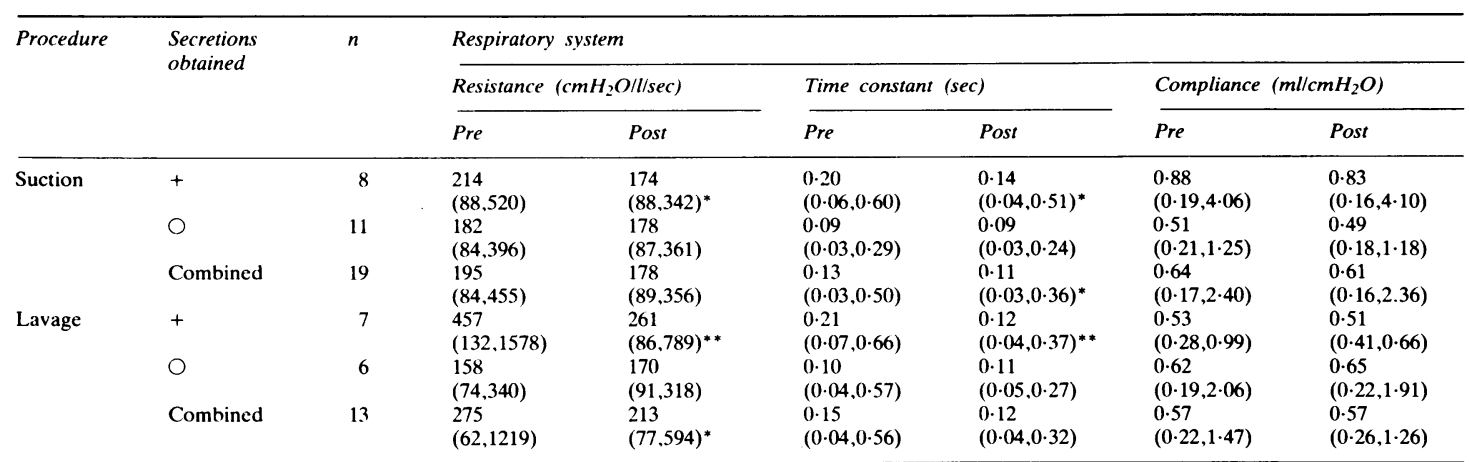

${ }^{*} \mathrm{p}<0 \cdot 05,{ }^{* *} \mathrm{p}<0 \cdot 01$, by $t$ test. Other differences not significant. Log values of respiratory system resistance, time constant, and compliance were used in the statistical analysis. 


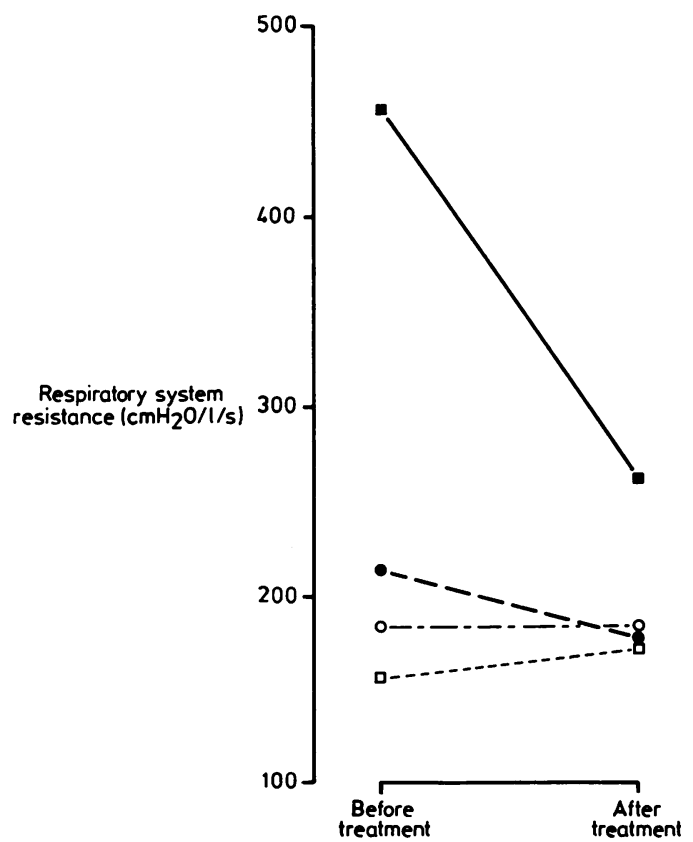

Fig. 1 Changes in respiratory system resistance after suction (circles) and lavage (squares) according to whether secretions were obtained (closed symbols) or were not obtained (open symbols). There was a significant reduction in resistance when secretions were obtained by lavage $(p<0.01)$ or suction $(p<0.05)$.

tendency for resistance to increase. Although there was a tendency for compliance to fall slightly after both procedures, no change was significant.

During the periods of continuous monitoring pronounced and consistent individual increases in resistance were occasionally seen, responding to tracheobronchial lavage (Fig. 2). In one individual an alarming increase in resistance indicated an urgent need to change a blocked endotracheal tube (Fig. 3).

\section{Discussion}

The adaptation of the single breath technique described by Zin $e t a l^{7}$ has provided a non-invasive means of monitoring the total respiratory system resistance. We have used this method to assess the timing and efficacy of routine tracheobronchial suction and lavage. Both of these procedures were effective in reducing resistance in patients whose resistance was abnormally high and in patients from whom visible secretions were removed by the procedures.

To obtain reference values for resistance in

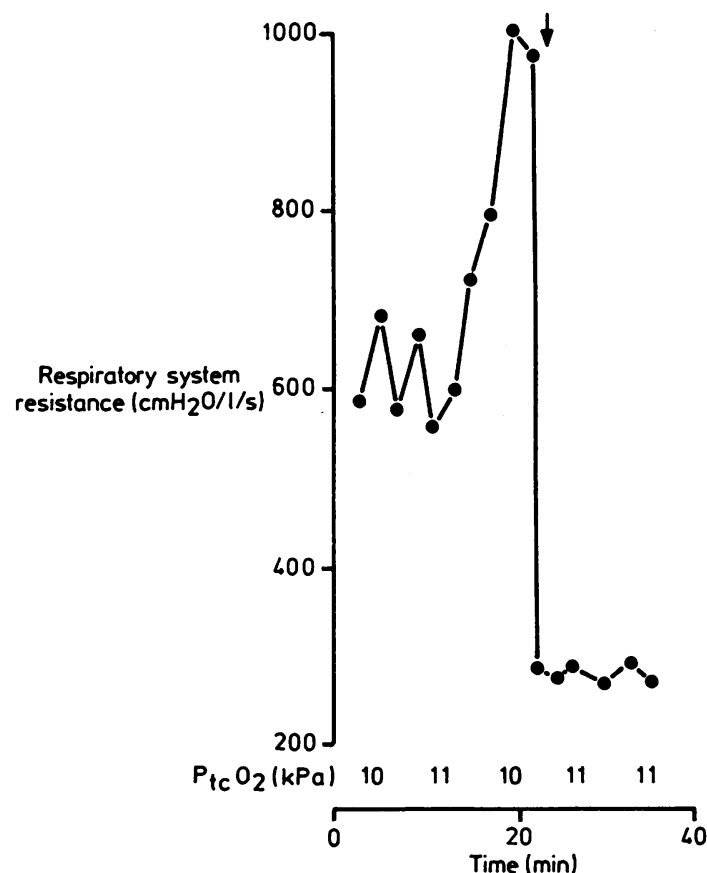

Fig. 2 Changes in respiratory system resistance and transcutaneous oxygen tension $\left(\mathrm{P}_{t \mathrm{C}} \mathrm{O}_{2}\right)$ in one infant during 35 minutes of continuous monitoring. A sudden increase in resistance was restored to normal by bronchial lavage (indicated by arrow).

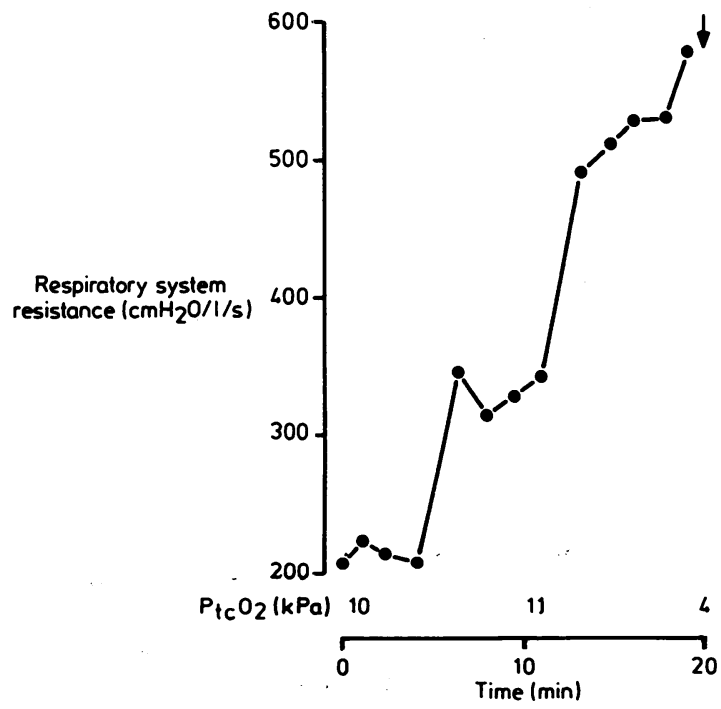

Fig. 3 Progressive rise in respiratory system resistance, and transcutaneous oxygen tension $\left(\mathrm{P}_{t c} \mathrm{O}_{2}\right)$ in one infant during 20 minutes of continuous monitoring. When removed (indicated by the arrow) the endotracheal tube was found to be completely occluded by mucus. 
intubated babies we studied a mixed group of mechanically ventilated, preterm infants over the first two days of life. The mean values of resistance were remarkably similar for infants with hyaline membrane disease and those with no respiratory tract disease and were close to the few published values for infants of this size. ${ }^{8-10}$ While it is clear that smaller babies (with smaller airways and smaller endotracheal tubes) must have higher values of resistance than larger infants, the range of ages of infants in our reference sample was not large enough to provide size related values. Nor has any attempt been made to subtract endotracheal tube resistance as the tube is functionally a very important and easily obstructed part of the infant airway. The 'correction' for tube resistance would in any case be complex as tube resistance is flow dependent. Interestingly, despite this pronounced change in tube resistance with flow, in most cases the end expiratory flow-volume curves were linear, implying a single value for the time constant. It is known that the airways resistance tends to increase as the lungs deflate. ${ }^{11}$ By coincidence, it seems that this effect may be balanced by the reduction in endoctracheal tube resistance as flow rates diminish during expiration.

By taking babies in their first two days of life, all of necessity older than $12-15$ hours, as the reference population, we hoped to control for all the variables that might affect resistance, with the exception of airway secretions, which appear clinically to be unusual during the first two days of mechanical ventilation in preterm infants. The values of resistance obtained provided a useful basis for predicting the benefits of suction or lavage and in the babies who did require such treatment an increase in resistance was usually noted before changes in arterial oxygenation (Fig. 2), although it is possible that arterial carbon dioxide tension might have been a more useful indicator of decreased alveolar ventilation. We attribute the late changes in oxygen tension, even with quite large increases in resistance, to the very short expiratory time constants found in these babies.

In babies with airways disease this technique of measuring respiratory resistance has several disadvantages. Firstly, all babies with external air leaks such as pneumothoraces had to be excluded from analysis. Two babies were found to have leaks around their endotracheal tubes, as indicated by their failure to maintain an inspiratory volume plateau. Their data were also excluded from analysis. Secondly, this system could not be used for more than four continuous hours in the ventilator circuit because of condensation in the ventilator tubing, which affected the accuracy of the pneumotacho- graphs. Thus continuous monitoring of resistance was not possible. Thirdly, in a small number of babies the relation between flow and volume between 50 and $97 \%$ of expired volume was not linear and therefore it was not possible to give a single value to resistance. It may be possible, however, to obtain some indication of respiratory resistance in such babies by observing the shape of the curve. Abnormally high resistance would be indicated by a flow-volume curve with a convex configuration towards the origin.

A major disadvantage is the need for passive expiration. Without oesophageal monitoring it may be impossible to exclude completely all respiratory effort. A linear expiratory flow-volume curve goes some way to confirming relaxation in intubated babies. The procedure that we sometimes used of hyperventilation to achieve apnoea by hypocapnia might affect resistance by altering bronchomotor tone. ${ }^{12}$ This effect is likely to be trivial compared with the resistance changes due to secretions and their treatment. In spite of these potential drawbacks, we were able to make reproducible baseline measurements of lung mechanics using the single breath technique in babies with severe degrees of airways obstruction and to show changes after tracheobronchial toilet. Others have provided in formation using the same technique on the changes in resistance after extubation in the neonata period. ${ }^{10}$

The benefits of tracheobronchial toilet were not universal. A balance is required between the potential benefits of removal of secretions (improved overall mechanical ventilation and more even distribution of ventilation) and the potential adverse affects (hypercapnia and hypoxia resulting from temporary ventilator disconnection, fall in compliance due to removal of positive pressure, and displacement of secretions into peripheral airways). When secretions were not obtained there was no change in resistance. One explanation is that the procedure itself was ineffective. This is unlikely as the mean value of resistance in the groups from whom no secretions were obtained after suction or lavage (Table 2) was close to the reference range. It seems likely, therefore, that these infants did not require 'routine' suction or lavage, with its potential adverse effects.

The level of resistance, in relation to a reference population, was a useful way of identifying patients with appreciable airway obstruction. After treatment resistance was almost halved in those with pretreatment values above the range of reference group. The change in time constant values was equally striking and of great clinical importance in this mechanically ventilated group. 
The time constant indicates the rate of emptying of the lungs, assuming exponential flow. Thus the shorter the time constant the more rapidly expiration is completed. The value of the time constant is therefore vitally important during mechanical ventilation; a long time constant due to mucous obstruction, for instance, may cause air trapping during rapid frequency ventilation if the expiratory time is short.

The group with the most severe airways obstruction (Table 1) had a mean time constant of 0.30 seconds. The extent of passive expiration depends only on the time constant and duration of expiration: $97 \%$ of the total volume is exhaled in $3 \times$ time constant. For infants with a time constant of over 0.30 seconds expiratory times of under 0.9 seconds may lead to air trapping. The current fashion for higher rates of mechanical ventilation might be hazardous in such patients. The fall in mean time constant to $0 \cdot 12$ seconds after tracheobronchial toilet was another measure of the success of the procedure.

There were no significant changes in compliance after any procedure. This is reassuring as both ventilator disconnection (by reducing the inflation pressure on the lungs) and displacement of secretions into peripheral parts of the lung might be expected to result in a fall in compliance. As compliance did not change and we made our measurements before and after suction under identical ventilator settings we assume that lung volume was also identical.

Potentially, continuous monitoring of resistance could obviate the sorts of crisis illustrated in Figure 3 . The ideal equipment does not exist for measuring endotracheal tube flow, although it may be possible to obtain an index of resistance without inserting any device into the flow pathway. ${ }^{13}$ We have indicated the potential advantages of continuous assessment of respiratory system resistance and the efficacy of a simple technique of tracheobronchial toilet. In the future a more satisfactory system for the long term monitoring of lung mechanics during mechanical ventilation may provide the basis for comparing different techniques of tracheobronchial care.
We thank Miss Sally Green, Mr Tim Demis, Mr Norman Levy, and Mr Jimmy Messeguer for technical help. The work was supported by grants from Action Research-The National Fund for Research Into Crippling Diseases, the London University Central Research Fund, and the Hammersmith and Queen Charlotte's Hospitals' Special Health Authority.

\section{References}

1 Storm W. Transient bacteremia following endotracheal suctioning in ventilated newborns. Pediatrics 1980;65:487-90.

2 Periman JM, Volpe JJ. Suctioning in the preterm infant: effects on cerebral blood flow velocity, intracranial pressure and arterial blood pressure. Pediatrics 1982:72:329-34.

3 Landa JF, Nwoke MA. Chapman GA. Brito M. Sackner MA. Effects of suctioning on mucociliary transport. Chest 1980;77: 202-7.

${ }^{4}$ Woodside NH, Latham SB, Denas SM. Increased recovery of neutrophils, macrophages and lymphocytes following repeated lavage of sheep lung in vivo. Exp Lung Res 1983:5:295-303.

5 Wong YC, Beardsmorc CS, Stocks J, Silverman M. Bronchial hypersecretion in preterm neonates. Arch Dis Child 1982: 57:117-22.

6 Fox WF, Schwartz JG, Schaffer TH. Pulmonary physiotherapy in neonates. Physiologic changes and respiratory management. $J$ Pediatr 1978:92:977-81.

${ }^{7}$ Zin WA, Pengally LD, Milic-Emili J. Single breath method for measurement of respiratory mechanics in anaesthetized animals. J Appl Physiol 1982;52:266-71

* Thomson A, Silverman M. Single breath measurement of lung mechanics in very low birthweight infants. Crit Care Med 1985;13:4-8.

${ }^{4}$ Le Soucf PN, England SJ, Bryan AC. Passive respiratory mechanics in newborns and children. Am Rev Respir Dis 1984:129:552-6.

1" Le Soucf PN, England SJ, Bryan AC. Total resistance of the respiratory system in preterm infants with and without an endotracheal tube. J Pediatr 1984;104:108-11.

1 Stocks J, Thomson A, Wong YC, Silverman M. The numerical analysis of pressure flow curves in infancy. Pediatric Pulmonology 1985:1:19-26.

12 Newhouse MT, Becklake MR, Macklem PT, McGregor M. Effect of alterations in end tidal $\mathrm{CO}_{2}$ tension on flow resistance. J Appl Physiol 1964;19:745-9.

${ }^{13}$ Mortola JA, Noworaj A. Two-sidearm tracheal cannula for respiratory airflow measurements in small animals. J Appl Physiol 1983;55:250-3.

Correspondence to Dr M Silverman. Department of Paediatrics and Neonatal Medicine, Hammersmith Hospital, Du Cane Road, London W12 OHS.

Received 30) April 1986 